\title{
A GPS sensing strategy for accurate and energy-efficient outdoor-to-indoor handover in seamless localization systems
}

\author{
Yungeun Kim, Songhee Lee, Seokjoon Lee and Hojung Cha* \\ Department of Computer Science, Yonsei University, Yonsei, Korea
}

\begin{abstract}
Indoor localization systems typically locate users on their own local coordinates, while outdoor localization systems use global coordinates. To achieve seamless localization from outdoors to indoors, a handover technique that accurately provides a starting position to the indoor localization system is needed. However, existing schemes assume that a starting position is known a priori or uses a naïve approach to consider the last location obtained from GPS as the handover point. In this paper, we propose an accurate handover scheme that monitors the signal-to-noise ratio (SNR) of the effective GPS satellites that are selected according to their altitude. We also propose an energy-efficient handover mechanism that reduces the GPS sampling interval gradually. Accuracy and energy efficiency are experimentally validated with the GPS logs obtained in real life.
\end{abstract}

Keywords: GPS, seamless localization, outdoor-to-indoor handover

\section{Introduction}

The widespread use of smartphones has fostered diverse location-based services, such as life logging [1], geotagging [2], tour guide service [3], and Social Network Services (SNS) [4,5]. These location-based services need to cover both indoor and outdoor environments. GSM-based localization [6,7] is one possible solution, but the scheme provides coarse-grained location information. GPS [8] has been a favorable locating solution in outdoor environments, but GPS signals are normally blocked by exterior walls. Therefore, many localization systems have been proposed to cover indoor environments. Using distance measurements between radios, UWB localization systems [9] provide precise locating and tracking services in indoor environments. However, the scheme is not adequate for locating smartphone users, as additional UWB tags and anchors are required. A bluetooth indoor positioning system [10] is also proposed but the system works only when there are enough number of stationary bluetooth devices.

The Wi-Fi Positioning System (WPS) [11,12] estimates the user's current location by finding the best matching data in the location database with current Wi-Fi scan results. WPS does not require additional infrastructure, but the scheme requires a costly and time-consuming offline training phase where a set of Received Signal Strength (RSS) from access points are collected at all interesting locations. The Pedestrian Dead Reckoning (PDR) [13,14] system is an alternative solution for locating smartphone users

\footnotetext{
${ }^{*}$ Corresponding author: Hojung Cha, Department of Computer Science, Yonsei University, Yonsei, Korea. E-mail: hjcha@ yonsei.ac.kr; hjcha@cs.yonsei.ac.kr.
} 
in indoor environments. The system tracks the user's moving path by using only inertial sensors such as the accelerometers, gyroscopes, and magnetometers used in off-the-shelf smartphones. The PDR system typically performs step-counting by monitoring the change of sensing values from an accelerometer, and it estimates moving direction by using a gyroscope and a magnetometer. Because the PDR system locates the user on its own local coordinates system, the scheme is commonly integrated with GPS. In this scenario, a handover process is necessary to trigger the PDR system whenever a smartphone user enters a building. To achieve high accuracy and efficiency of the PDR system, the handover process should consider the following issues. First, the process should trigger the PDR system as late as possible, as the accuracy of the PDR system decreases as time advances, due to drift error. Second, the handover process should trigger the PDR system before GPS becomes inaccurate or unavailable, as the PDR uses the last GPS location as a starting position.

There have been several seamless localization systems that used additional anchors or devices to perform accurate handover [15-19]. However, those approaches are not practical for smartphone users. Hansen et al. [20] proposed a handover technique that uses only GPS. In this system, handover is performed when the GPS signals are blocked completely, and the last location from GPS is considered the handover position. This approach is problematic, as GPS still provides an inaccurate location for a while, even after the user enters a building.

In this paper, we propose a handover technique that monitors the signal-to-noise ratio (SNR) of GPS satellites. We observed that there is a short period of time when the SNR of GPS satellites drops rapidly, while accurate location information is still provided, near the entrance to a building. We also found that satellites positioned behind the user, at high altitudes, are more effective for finding the most appropriate handover time. The proposed method first selects effective satellites based on altitude, then finds the most appropriate handover time when the SNR of the selected GPS starts to drop. The proposed method achieves high accuracy, but large energy consumption is inevitable due to the continuous GPS sampling. We overcome this problem by gradual increment of the GPS sampling rate over several visits to the Point of Interest (POI). Initially, a low rate of GPS sampling is used, and the reference point is produced where the GPS signal has been cut off. Then, the error bound of the reference point shrinks, at each revisit, to the reference point by gradual increments of the GPS sampling rate. Finally, the proposed handover technique is performed when the error is small enough to guarantee energy efficiency. The assumption of revisiting is reasonable since people tend to visit several POIs repetitively in their normal life. The contributions in this paper are as follows:

- We propose an accurate outdoor-to-indoor handover technique that works by monitoring the SNRs of GPS satellites without additional infrastructures. We also reveal that GPS satellites positioned behind the user and at high altitudes are more sensitive to movement from outdoors to indoors than other satellites.

- We significantly reduced energy consumption by gradual increments of the GPS sampling rate. An accurate handover position can be acquired by several re-visits.

- With the proposed method, the handover is always performed around the entrance to a building. In other words, the handover positions acquired from the proposed method can be exploited to investigate the layout of the building.

The remainder of this paper is structured as follows. Section 1 discusses the related works on heterogeneous localization systems. Section 2 analyzes the characteristics of GPS when a user enters a building. Section 3 describes a handover method that exploits the SNRs of specific GPS satellites. In Section 4, we present an energy-efficient method that increases the GPS sampling rate gradually, followed by an evaluation with real experiments in Section 5. We conclude the paper in Section 6. 


\section{Related work}

In recent years, there has been active research to provide location services for both indoor and outdoor environments. Beauregard et al. [15] proposed a combined approach of GPS and PDR. The system tracks the user by using GPS in the outdoor environment and triggers the PDR when the GPS becomes unavailable in the indoor environment. The PDR tracks the user by estimating step length, step count, and heading with inertial sensors. Chon et al. [1] proposed a life-logging system that also combines GPS and PDR. They used inertial sensors built into smartphones for PDR in indoor environments. Kourogi et al. [16] proposed an approach that combines GPS, PDR, and RFID. The system improved the accuracy of the PDR with an active RFID tag system sparsely placed in key areas. Because PDR is a relative navigation system, the handover from outdoors to indoors is an important issue in these systems. Skyhook Wireless [17] proposed a commercial system that covers both indoor and outdoor environments using a Wi-Fi fingerprinting technique. The system built up a huge database of radio signal strength in the entire region by driving a car equipped with GPS and a Wi-Fi device. The system showed poor accuracy in indoor environments, as war-driving surely cannot access indoor environments. Hansen et al. [18] proposed a combined approach of GPS and the Wi-Fi fingerprinting system. They presented four solutions for the handover between GPS and Wi-Fi: preferring GPS, preferring Wi-Fi, preferring GPS until the signal is lost, and preferring GPS upon continuous readings. They evaluated the applicability of the solutions with respect to accuracy and energy consumption. Using Wi-Fi fingerprinting, however, has a practical problem: An offline training phase is required to build a radio map of every indoor environment. Several approaches have combined GPS and UWB [19,20], which provides accurate range estimation in high multi-path indoor environments. These approaches extend the capability of localization in hostile environments by using UWB to augment GPS, but they require costly deployment of UWB devices to a target area and a UWB tag in the target device.

As location-based services are available to smartphone users, active research has been conducted on the energy-efficient localization systems on smartphones. Micro-blog [21] is a participatory sensing system that allows smartphone users to share geo-tagged multimedia. The system achieves a good energyaccuracy tradeoff by switching between different localization schemes such as WPS, GSM localization, and GPS dynamically. EnTracked [22] tracks mobile devices in an energy-efficient way that schedules the position update based on the estimation and prediction of system conditions and mobility. The system also considers the delays for powering the GPS on and off in order to achieve robustness. EnLoc [23] is an energy-efficient localization system that uses personal profiles for mobility patterns. In this system, the user's habitual mobility pattern is profiled, and energy consumption is reduced by mobility prediction based on the profile. However, these approaches do not focus on the accurate and energy-efficient handover from outdoor to indoor environments.

\section{Characteristics of GPS when entering indoor environment}

Existing localization systems simply perform handover from outdoors to indoors when the GPS signal is cut. However, this approach is problematic, as the GPS signal is not blocked perfectly and, accordingly, accuracy becomes low when entering an indoor environment. We conducted a preliminary experiment to analyze the change of SNR and accuracy of GPS while entering a building. We sampled GPS signals with an Android smartphone, Nexus S, in front of three different buildings at Yonsei University, then estimated error distance by using Google Earth. 


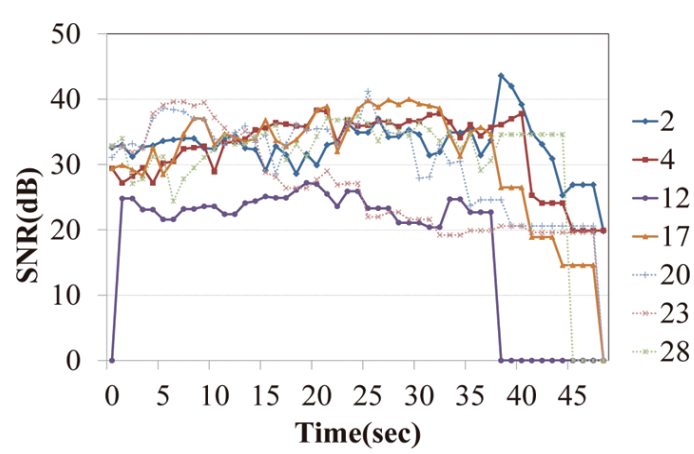

(a)

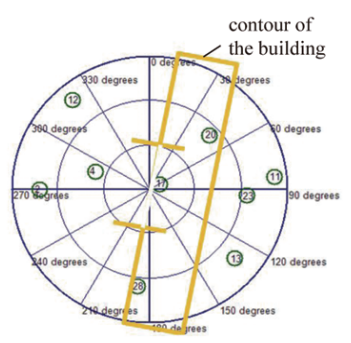

(b)

Fig. 1. Changes in SNR according to the GPS satellites when entering a building: (a) SNR changes when entering a building, and (b) the position and the altitude of satellites.
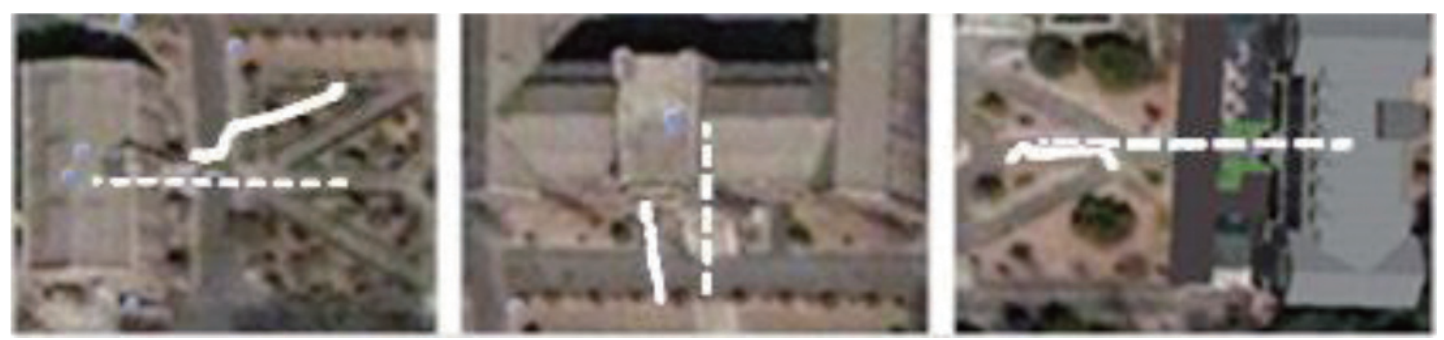

Fig. 2. GPS error near the entrance of the building. Dotted lines are real paths, and solid lines are GPS trajectories.

Figure 1(a) illustrates the changes in the SNR of GPS when entering a building. The vertical line represents the time when the user is at the entrance to the building. GPS signals were not blocked perfectly, but the SNR of GPS was reduced gradually after the user entered the building. The penetration of the GPS signal was retained for more than 10 seconds, and the number of GPS satellites was kept at more than four for $8-15$ seconds. This means that the signal cutoff is not a good criterion of handover point from outdoors to indoors. Moreover, the SNR change varied among different GPS satellites. SNRs of some satellites decreased rapidly right after entering the building, whereas SNRs of others decrease slowly. This is because the times when the line of sight (LOS) was blocked by the building differed, depending on the location of the GPS satellites, as shown in Fig. 1(b). Figure 2 illustrates the level of accuracy of GPS when entering a building from outdoors.

The solid line represents the path estimated by GPS, and the dotted line represents the ground-truth path. The solid lines are shorter than the dotted lines, which means that GPS estimated the user's location as outside of the building even after the user entered the building. This can be explained by the feature of localization method in GPS. GPS estimates location by trilateration, with information received from more than four satellites. The accuracy of trilateration is dependent on Geometric Dilution of Precision (GDOP), a condition of arrangement of GPS satellites. In locations far from buildings, high accuracy is achieved because GPS can receive signals from satellites that are scattered all over. However, near a building, signals from the satellites over the building are blocked by the building. As a result, the satellites used for trilateration are close together, and GPS accuracy becomes worse. When the signal from the satellite over the building is received, the distance from the satellite is misjudged as longer than it really is, due to the NLOS (Non-Line Of Sight). In this case, the result of the trilateration is outside of the building, as in Fig. 2. 


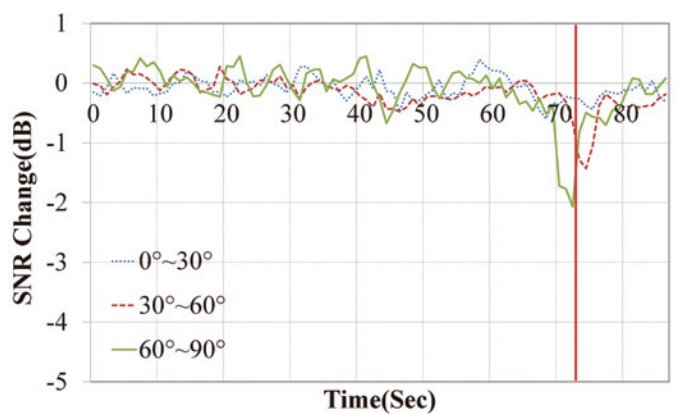

(a)

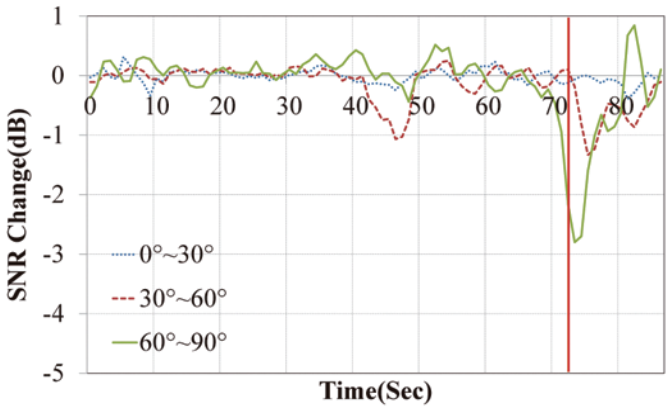

(b)

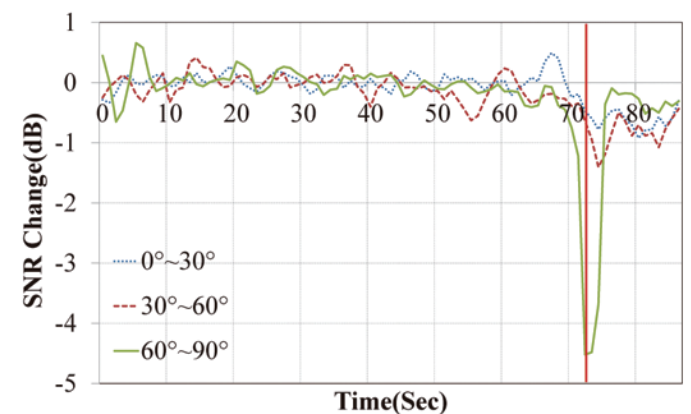

(c)

Fig. 3. SNR changes according to elevation: (a) SNR changes with HTC hero (b) SNR changes with HTC Nexus S (c) SNR changes of Samsung Galaxy S2. The vertical line represents the time when a user is at entrance.

In this experiment, we found that the GPS signal cutoff is not adequate for determining the handover point, as the GPS signal penetrates the building for a while after the user enters. Alternatively, we can exploit SNR changes to determine the handover point, as the SNRs of the specific satellites rapidly decrease around the building entrances. In the next section, we propose an accurate handover method that finds the time when the SNRs of the specific satellites start to drop but the GPS accuracy still remains high.

\section{Handover based on monitoring changes in SNR}

According to the preliminary experiment, the SNR of GPS starts to decrease rapidly near the entrance to the building. The average SNR dropped from $30 \mathrm{~dB}$ to lower than $20 \mathrm{~dB}$ after entering the building. However, the exact location of the entrance could not be determined by using a fixed value of average SNR as a threshold, as the degree of SNR reduction varied, depending on the environment around the building and the positions of the GPS satellites. Therefore, we propose a handover technique that monitors the changes in SNRs of the specific satellites rather than using a fixed level of SNR as a threshold. We first selected the GPS satellites that were expected to show significant SNR changes near the entrance to the building. We then determined the best time to perform the handover by monitoring the SNR changes in the selected satellites.

In the preliminary experiment, we looked for characteristics of the GPS satellites that showed significant SNR changes at the entrance of the building, and we found that these satellites float at high altitude. To validate the result, we conducted additional experiments. We sampled the GPS data while walking 


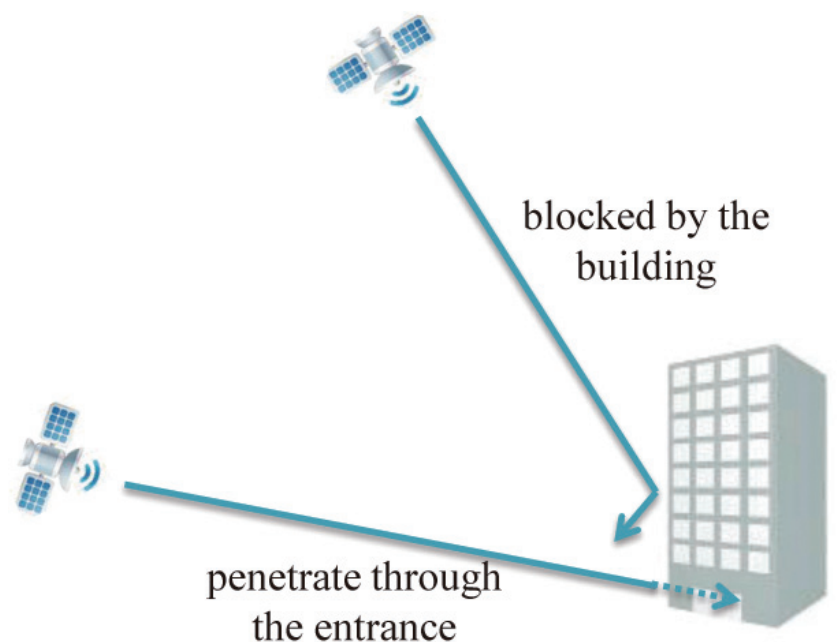

Fig. 4. The effect of the elevation of the satellite.

into three different buildings for 90 times (30 times per a building), and analyzed the SNR changes depending on the altitude of the satellites. For the experiment, three different smartphones, HTC Hero, HTC Nexus S, and Samsung Galaxy S2, are used to considering the issue on device diversity. As shown in Fig. 3, satellites at an elevation of 30-90 degrees showed a dip in SNR changes near the vertical line that represents the time when the user is at the entrance. However, satellites at an elevation of 0-30 degrees showed no noticeable SNR drop around the vertical line. This is because the signal from the high elevation was blocked by the building, whereas the signal from the low elevation could penetrate the building through the entrance, as illustrated in Fig. 4. The vertical line is near the center of dip where the SNR change is the maximum. In other words, we can find the entrance by detecting a dip in SNR changes.

To find an optimal handover position, we propose a selection algorithm in the following, based on the altitude of the satellites:

1. The GPS satellites are arranged in the order of altitude.

2. The $n$ highest satellites are selected for monitoring.

3. SNR changes are recorded while GPS is available.

4. When GPS is unavailable, find a dip in the series of SNR changes.

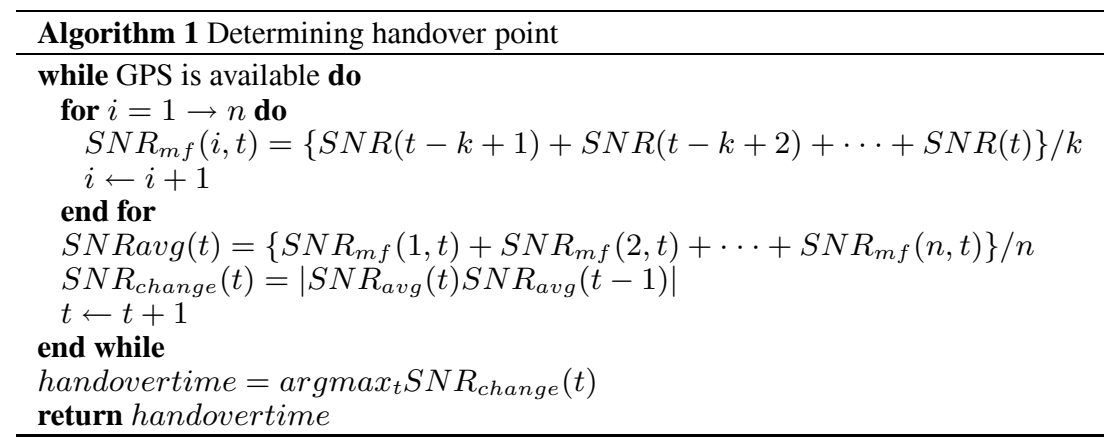

We selected three satellites ( $n=3$ ), a quarter of the average number of visible satellites in a certain location. 
Algorithm 1 explains the overall process of determining the handover point by monitoring the SNRs of the selected satellites. We continuously recorded SNR changes until GPS became unavailable inside the building. Then, the location where the greatest SNR change was observed was determined to be the handover point. The SNR was sampled every second, and a mean filter was applied to smooth the short-term variation of the SNR. The proposed algorithm has a drawback in that the handover point cannot be determined until GPS becomes unavailable. However, this is not a big issue if the PDR system can be turned on and record logs in advance. When GPS becomes unavailable later, the PDR system can recover the path based on the handover position and the recorded logs.

\section{Energy-efficient GPS sampling}

The proposed handover method requires a GPS sampling interval of one second, which may lead to significant power consumption. Because it is not practical to use a short sampling interval with a smartphone in normal life, many location-based services use GPS only once every 1-5 minutes. However, these location-based services cannot estimate the location precisely in indoor environments due to the long sampling interval. This is the tradeoff between accuracy and power consumption. In this section, we propose an energy-efficient method that reduces the GPS sampling interval gradually for POIs that are frequently revisited. The assumption of revisiting is acceptable according to the survey of Yohan et al. [24]. In the survey, people visit several primary places, such as home and office, repetitively, and the probability that people visit a new place is lower than $25 \%$.

Figure 5 illustrates the overall process. Initially, the user's path is continuously tracked at a sufficiently long sampling interval to save power consumption. When the user enters the indoor environment and GPS becomes unavailable, a disconnection point (DP) is created on the location acquired from the last GPS signal, and an interesting boundary (IB) is created around the DP. Each time the user enters the IB, the DP and the IB are revised by gradual reduction of the GPS sampling interval. When the IB shrinks to a small size to guarantee energy efficiency, the proposed handover technique is applied to find the entrance to the building. In this section, we describe in detail reducing the GPS sampling interval and additional power saving strategies after entering the IB.

\subsection{Gradual reduction of GPS sampling interval}

When a user visits a certain place in an indoor environment for the first time, the GPS sampling interval $P_{1}$ is set to $P_{0}$, which is a predefined initial sampling interval to track the smartphone user continuously. When GPS becomes unavailable, the DP and IB are created, as shown in Fig. 6. The location of the DP is determined by the last GPS information. Because we cannot know the exact disconnection time $E_{1}^{d p}$, the maximum error of the DP is calculated as Eq. (1):

$$
E_{1}^{d p}=V \times P_{1}
$$

$V$ represents moving speed of the user. Because exact moving speed is not required in this method, $V$ is roughly defined as the average walking speed of people. The IB is then created as a circle that has a radius calculated by Eq. (2):

$$
R_{1}^{i b}=E_{1}^{d p}+E^{e x}
$$




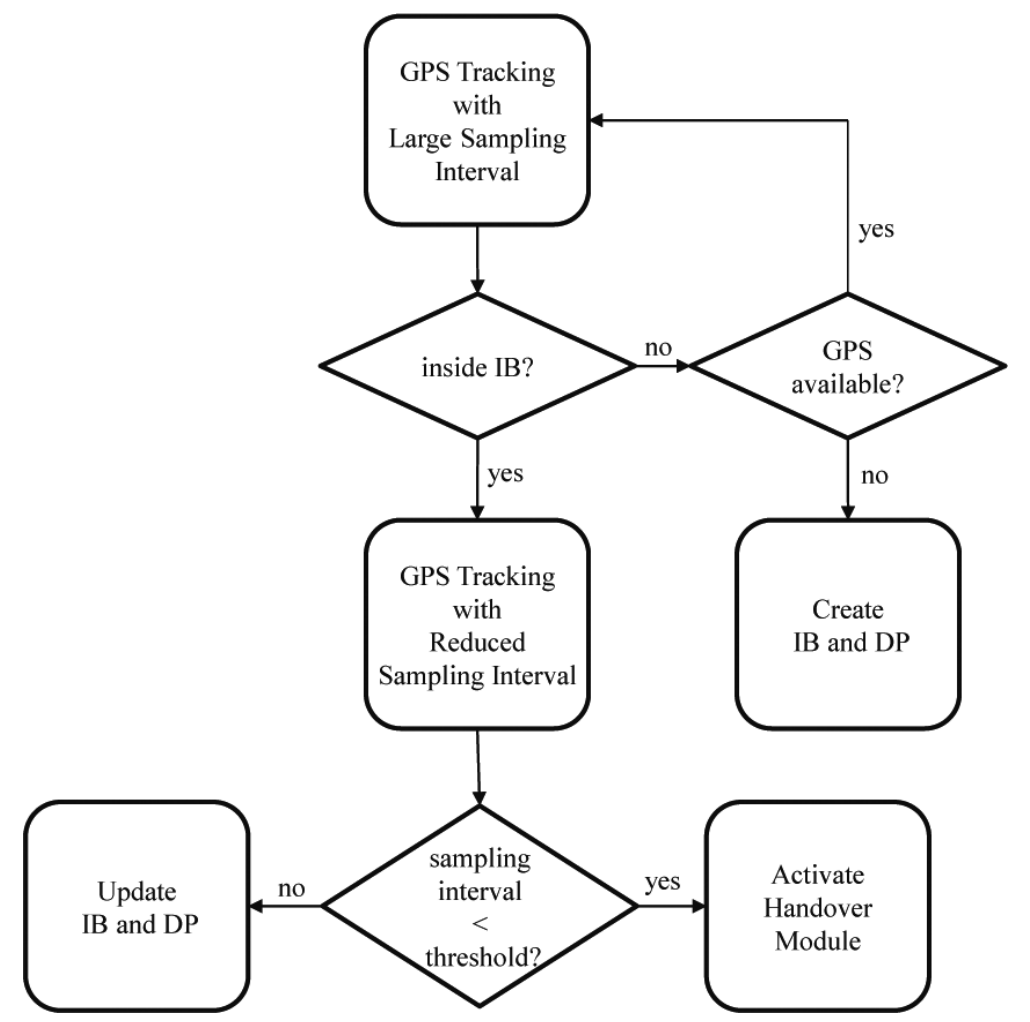

Fig. 5. Overview of the proposed energy-efficient method.

To prevent the user from entering the indoor environment without any GPS use, an extra boundary $E^{e x}$, calculated as Eq. (3), is added:

$$
E_{e x}=V \times P_{0}
$$

When the user enters the IB for the nth time, the GPS sampling rate increases as follows:

$$
P_{n}=P_{(n-1)} \times r,(n>1)
$$

where $\mathrm{r}$ represents the reduction factor. When GPS becomes unavailable, the error of the DP and the radius of the IB shrink as follows:

$$
\begin{aligned}
& E_{n}^{d p}=V \times P_{n} \\
& R_{n}^{i b}=E_{n}^{d p}+E^{e x}
\end{aligned}
$$

When $E_{n}^{d p}$ shrinks to threshold $E^{t h}$, which is small enough to guarantee energy efficiency, the handover technique proposed in Section 3 is applied the next time.

\subsection{Power-saving strategy inside IB}

We set an extra boundary $E_{\text {ex }}$ to guarantee at least one GPS sampling before entering the building. When the user is detected about to enter the IB, as shown in Fig. 7, using GPS in this boundary is wasteful, 

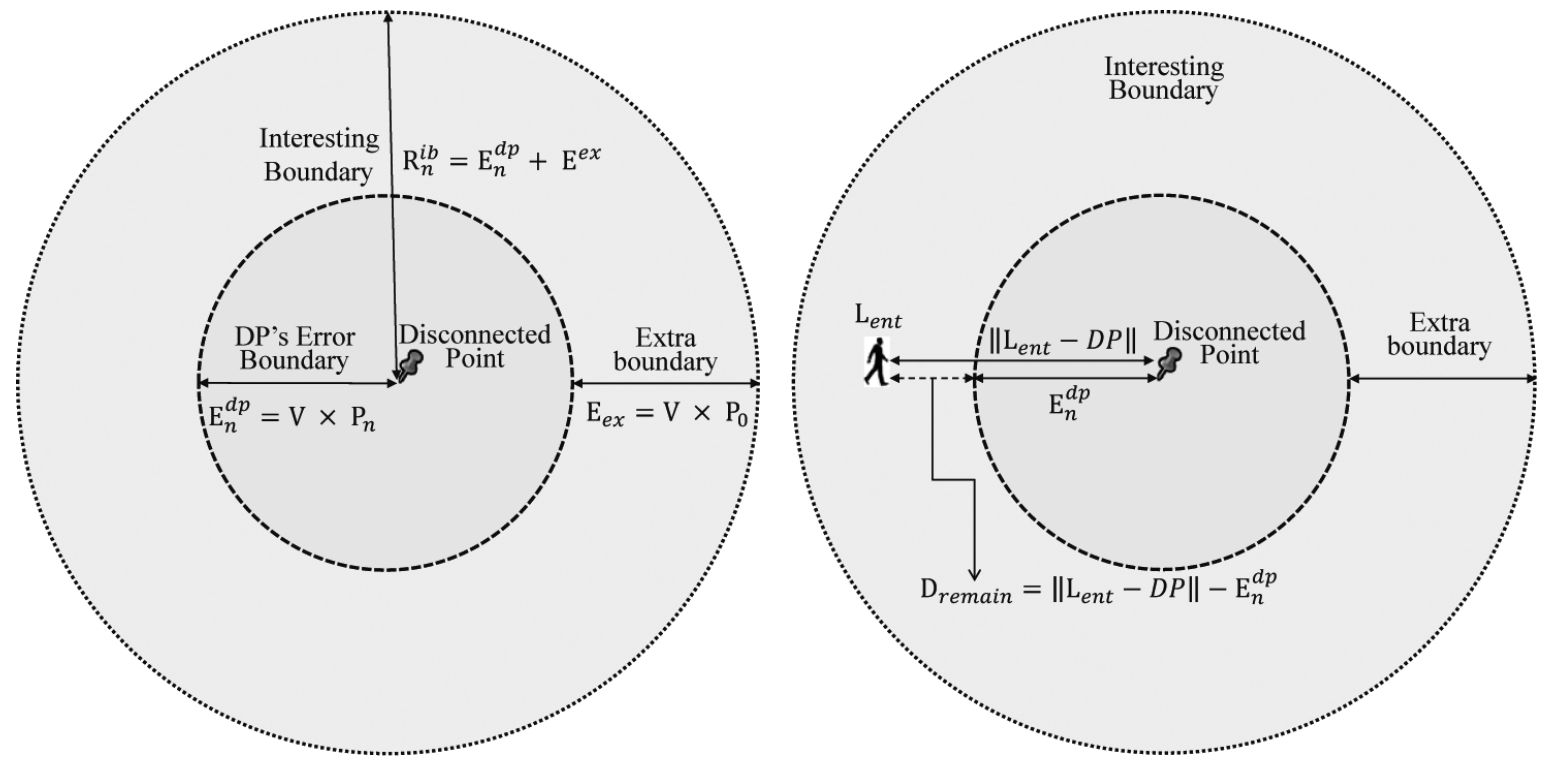

Fig. 6. Creation and update of the DP and IB at $n$th visit. Fig. 7. Method of reducing energy consumption inside the IB.

because we already know the maximum error of the DP where GPS starts to be sampled. Therefore, we additionally reduce power consumption by forbidding the use of GPS when the user is expected to be in an extra boundary.

When the user is detected inside the IB at location $L_{e n t}$, the remaining distance to the error boundary of DP is calculated as Eq. (7):

$$
D_{\text {remain }}=L_{e n t}-D P-E_{n}^{d p}
$$

Then, GPS sampling is delayed by the duration calculated as Eq. (8):

$$
T_{\text {delay }}=D_{\text {remain }} V
$$

This strategy is efficient when initial sampling rate $P_{0}$ is low and increment factor $r$ is high.

\section{Experimental results}

This section discusses the results of the experiments that were conducted to evaluate the proposed handover technique. First, the accuracy of the handover technique was validated at three different buildings. We then conducted a series of emulation experiments with GPS logs sampled in real life to evaluate the energy efficiency of the gradual increments of the GPS sampling rate. Finally, we evaluated the overall performance with a real experiment.

\subsection{Accuracy of the proposed handover technique}

To evaluate the proposed handover technique, we implemented the scheme on a NexusS Android smartphone and conducted experiments at three buildings with different characteristics, as shown in Fig. 8. The Engineering Hall showed a rapid SNR drop at the entrance, as it is a high-rise building with 


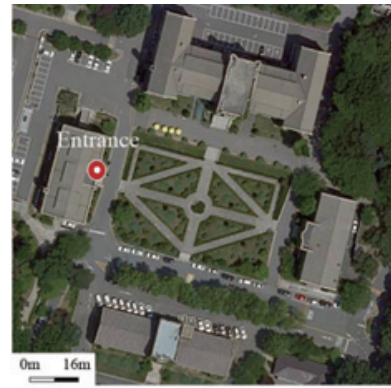

(i) Memorial Hall

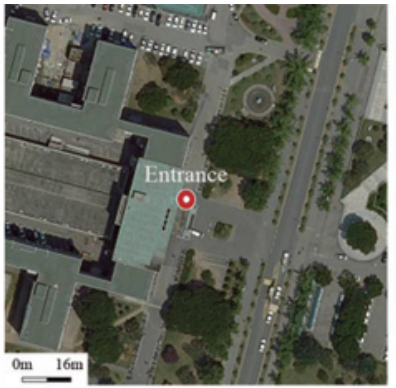

(ii) Engineering Hall

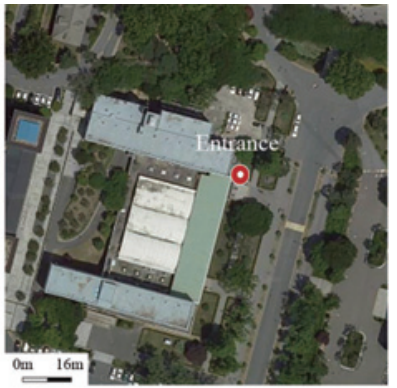

(iii) Baekyang Hall

(a)

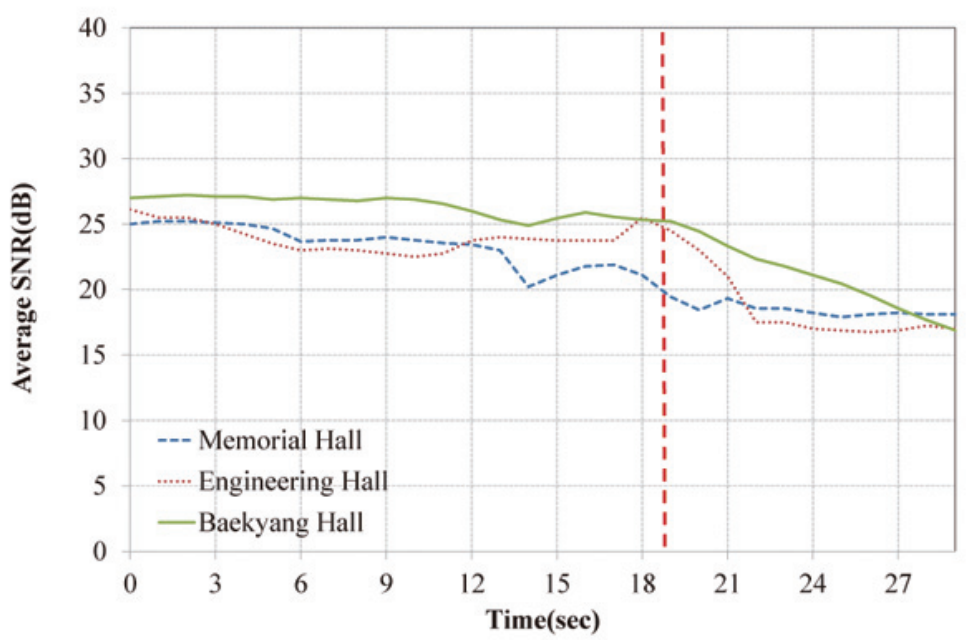

(b)

Fig. 8. Characteristics of the three different buildings. (a) Environment around the buildings, and (b) changes when entering the buildings.

six stories, around which there are no other obstacles. Baekyang Hall showed a slow SNR drop at the entrance because it has only two stories. Memorial Hall is surrounded by other buildings, so the SNR fluctuated near the building. We also considered the effect of the time period. To evaluate the algorithm in harsh conditions, we conducted the experiment from 16:30 to 18:00, when the number of visible GPS satellites is the smallest in the region. We compared the proposed technique (Selected SNR) with two other approaches: the All SNR method, which monitors the SNRs of all visible satellites, and the Signal cutoff method, which uses as the handover point the location where GPS becomes unavailable.

Prior to actual evaluation, we conducted a preliminary experiment that performed the proposed algorithm by changing the window size. A small window size is vulnerable to short-term noise, whereas a large window size is insensitive to environmental changes from outdoors to indoors. We eventually decided to use a window size of three, which estimated the handover point the best, as shown in Table 1 .

Figure 9(a) illustrates the time error between the handover point and the time when the user entered the building. Selected SNR always performed the handover within five seconds, whereas the other methods showed a time error greater than 10 seconds, depending on the building. As shown in Fig. 9(b), the error distances of the proposed algorithm were $5.2 \mathrm{~m}, 2.6 \mathrm{~m}$, and $8.9 \mathrm{~m}$ in Memorial Hall, Baekyang Hall, and the Engineering Hall, respectively, while the error distances of All SNR were $13.2 \mathrm{~m}, 5.3 \mathrm{~m}$, and $17 \mathrm{~m}$, 
Table 1

The effect of the window size of mean filter

\begin{tabular}{ccc}
\hline Window size & Average error distance & Standard deviation \\
\hline 1 & $6.57 \mathrm{~m}$ & $5.34 \mathrm{~m}$ \\
3 & $5.59 \mathrm{~m}$ & $2.69 \mathrm{~m}$ \\
5 & $5.88 \mathrm{~m}$ & $3.07 \mathrm{~m}$ \\
7 & $5.82 \mathrm{~m}$ & $3.39 \mathrm{~m}$ \\
9 & $5.69 \mathrm{~m}$ & $2.96 \mathrm{~m}$ \\
11 & $5.72 \mathrm{~m}$ & $2.96 \mathrm{~m}$ \\
\hline
\end{tabular}

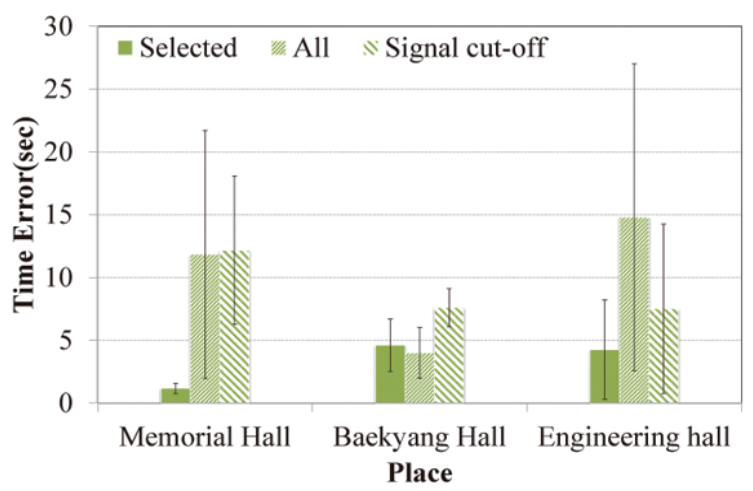

(a)

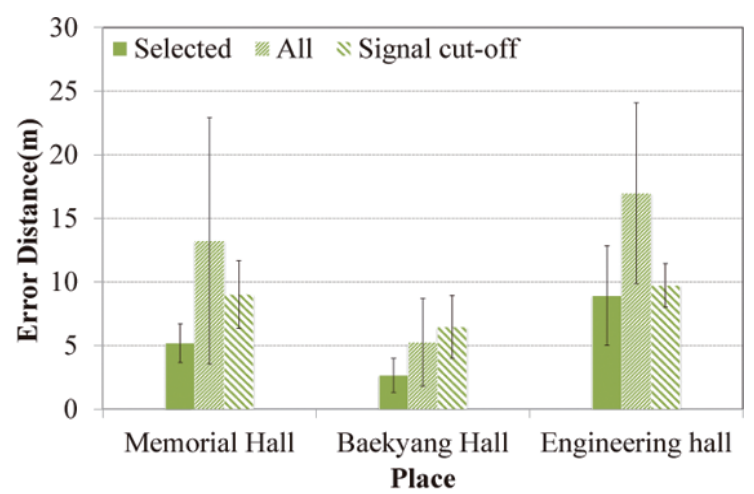

(b)

Fig. 9. Handover accuracy according to the three different methods. (a) Time error of the handover point, and (b) Error distance of the handover point.

and Signal cutoff showed error distances of $9.0 \mathrm{~m}, 6.5 \mathrm{~m}$, and $9.7 \mathrm{~m}$. Selected SNR showed a smaller error distance than the others, regardless of the building, whereas All SNR showed a worse result than Signal cutoff. This means that the proposed algorithm selected only influential satellites and monitored the SNRs successfully to estimate the building entrances.

\subsection{Energy efficiency of the gradual reduction of GPS sampling intervals}

To evaluate the proposed method, we should define $E^{t h}$, the threshold for activating the handover. Because the algorithm does not consider the error distance of the GPS, the threshold must be large enough to cover the GPS error near the building. We measured the GPS error from a location $30 \mathrm{~m}$ away from the Engineering Hall to the entrance 20 times. We determined $10 \mathrm{~m}$ as the value of $E^{t h}$, as $90 \%$ of the error was smaller than $10 \mathrm{~m}$, as shown in Fig. 10.

We then conducted a simulation with GPS logs sampled in the real environment. We collected GPS logs per second while walking to the Engineering Hall from the location $1 \mathrm{~km}$ away from the entrance. The initial sampling interval $P_{0}$ was set to five minutes. The simulation was conducted by changing the time of the first GPS sampling randomly.

Because the performance of the proposed method depends on the reduction factor $r$, we simulated the method by changing $r$.

Figure 11 shows the required number of visits to activate the handover and the overall energy consumption according to $r$. As $r$ decreases, the required number of visits decreases and, consequently, the energy consumption is also reduced. The rapid drop in sampling intervals leads to frequent GPS sampling in the extra boundary, where GPS tracking is useless for handover. The proposed algorithm 


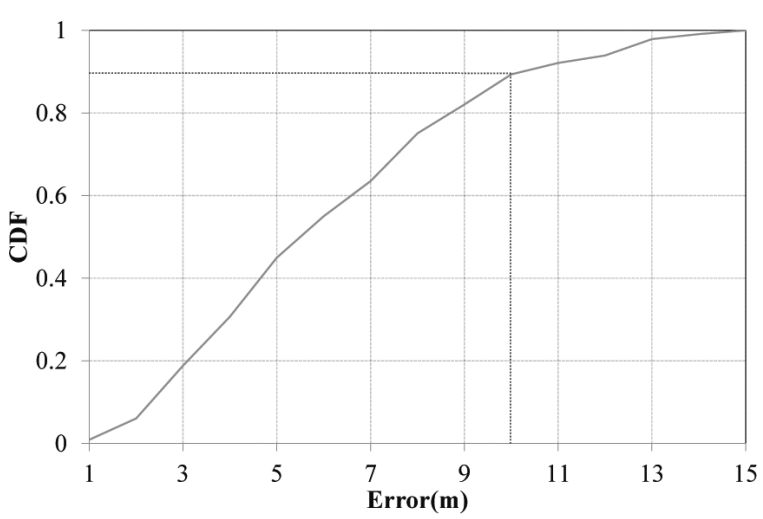

Fig. 10. CDF of the GPS error near the building.

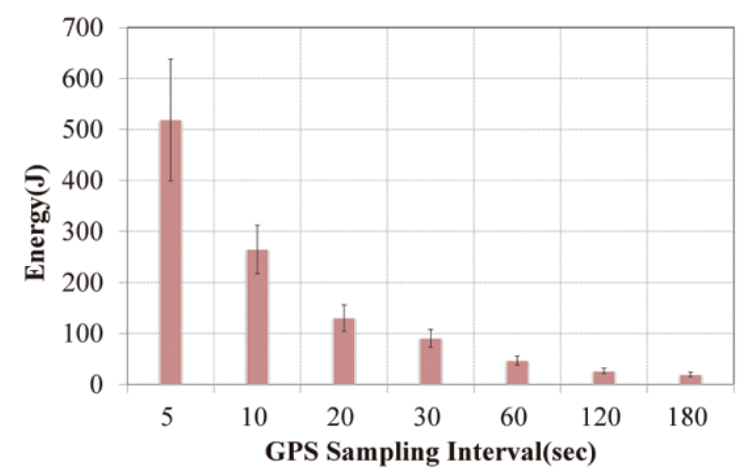

(a)

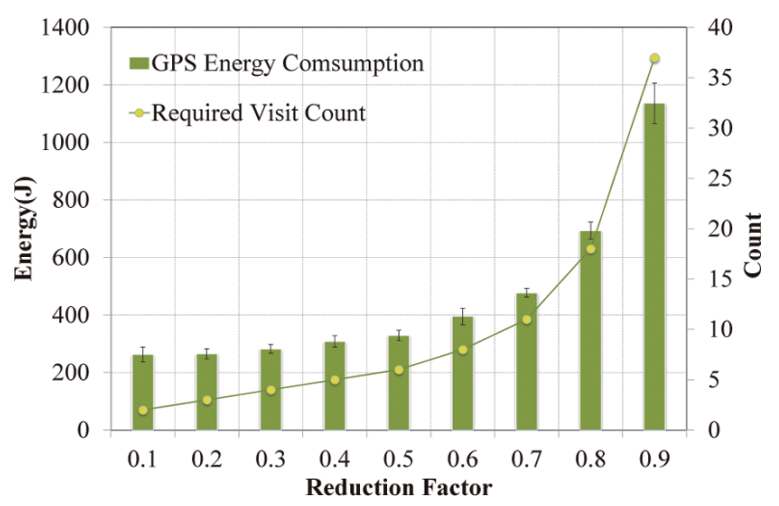

Fig. 11. Effect of reduction factor $r$.

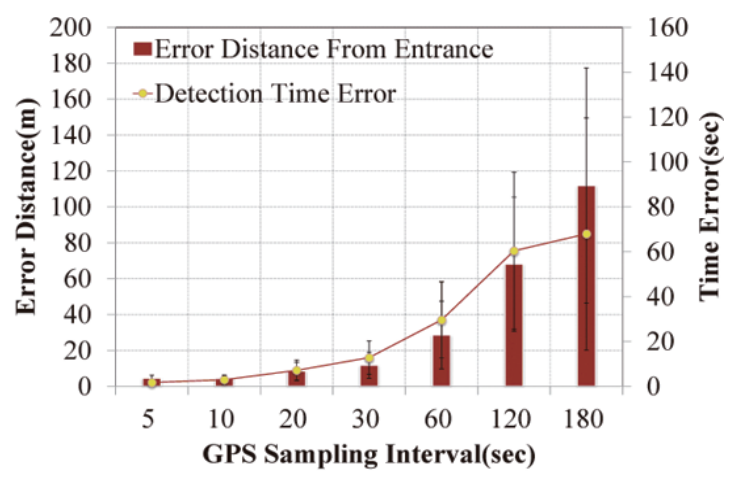

(b)

Fig. 12. Performance of the method of using fixed sampling interval. (a) Energy consumption vs. GPS sampling interval, and (b) Time and distance error vs. GPS sampling interval.

handled this problem by turning the GPS off in the extra boundary. The best result was when $r$ was 0.1 , where the handover method was activated on the third visit, with energy consumption of about $230 \mathrm{~J}$.

We also evaluated the performance of using a fixed GPS sampling interval, regardless of the number of visits.

Figure 12 illustrates the accuracy and energy consumption of the fixed sampling intervals of 5,10 , 20, 30, 60, 120, and 180 seconds. The distance error represents the GPS error of the handover point, and the time error represents the time difference between the handover point and the time when the user actually entered the building. As the sampling interval becomes shorter, the error distance and the time error become smaller, but the energy consumption increases. The result of the 10 seconds showed the error distance to be less than $5 \mathrm{~m}$, with $250 \mathrm{~J}$, and the result of the 20 seconds showed the error distance to be less than $10 \mathrm{~m}$, with $130 \mathrm{~J}$.

Finally, we compared the results of the proposed method with the results of these two fixed sampling intervals, which showed reasonable accuracy and energy consumption.

As shown in Fig. 13, the proposed method showed the best results for both accuracy and energy efficiency. The average energy consumption was $110 \mathrm{~J}$, which is less than that of the 20 seconds. The error distance was $4 \mathrm{~m}$, which is also smaller than that of the 10 seconds. The average time error of the proposed method was about 1.5 seconds, much less than those of the fixed sampling intervals, which means that the proposed method accurately performed handover near the entrance. 


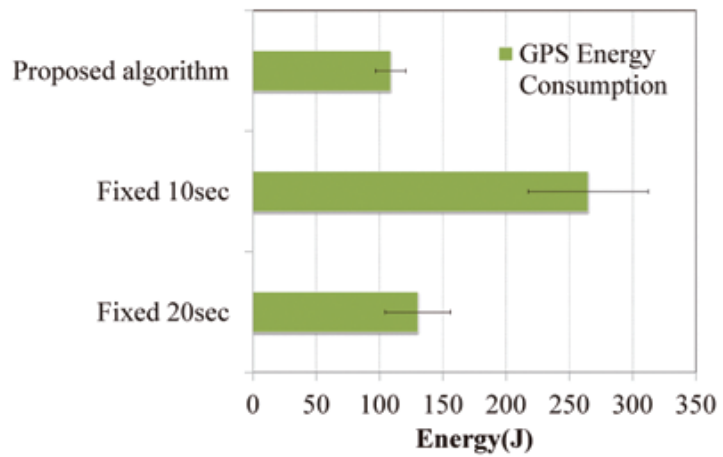

(a)

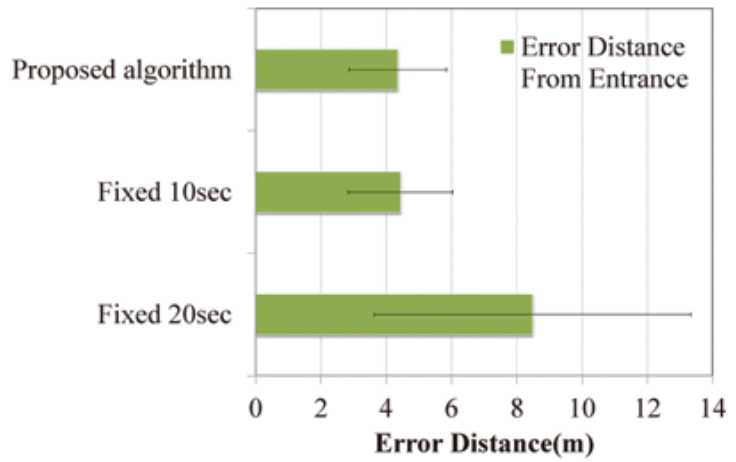

(b)

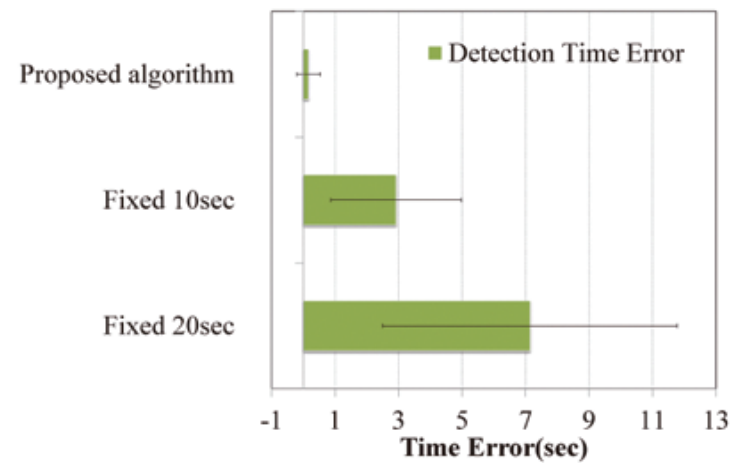

(c)

Fig. 13. Performance of the three different GPS sampling methods. (a) energy consumption, (b) error distance, and (c) time error.

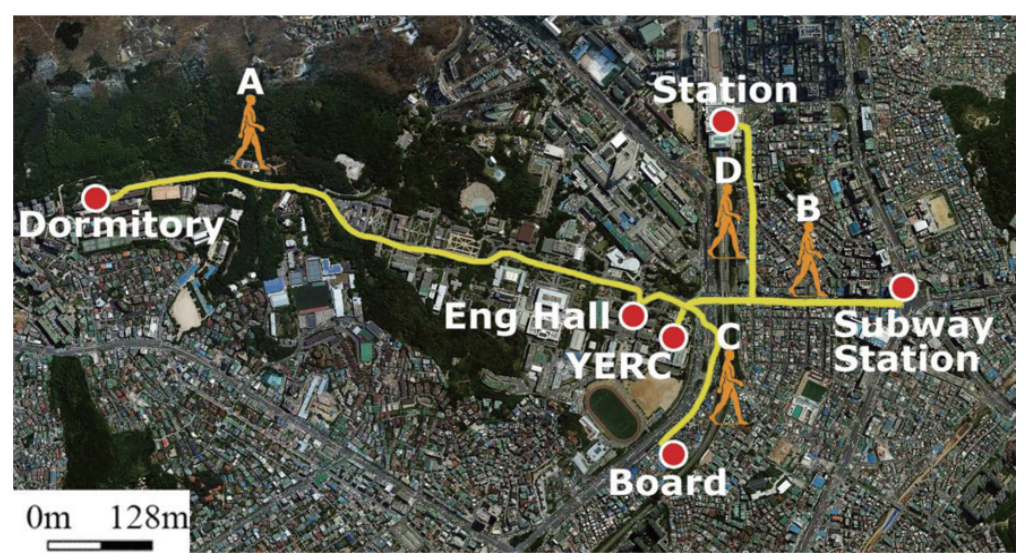

Fig. 14. Paths of the users in the real-life experiment.

\subsection{Evaluation with a real-life experiment}

We conducted experiments to evaluate the proposed method in real life. Four participants conducted the experiment with four different paths as shown in Fig. 14.

Participant A departed for the Engineering Hall from the dormitory in the morning and vice versa 


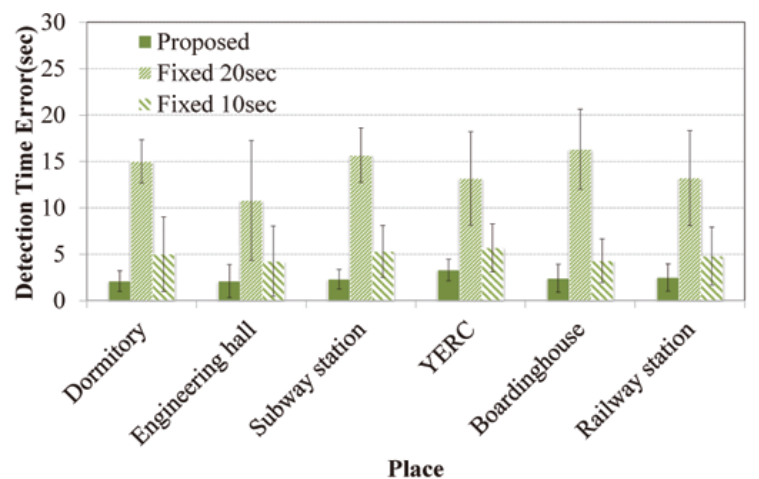

(a)

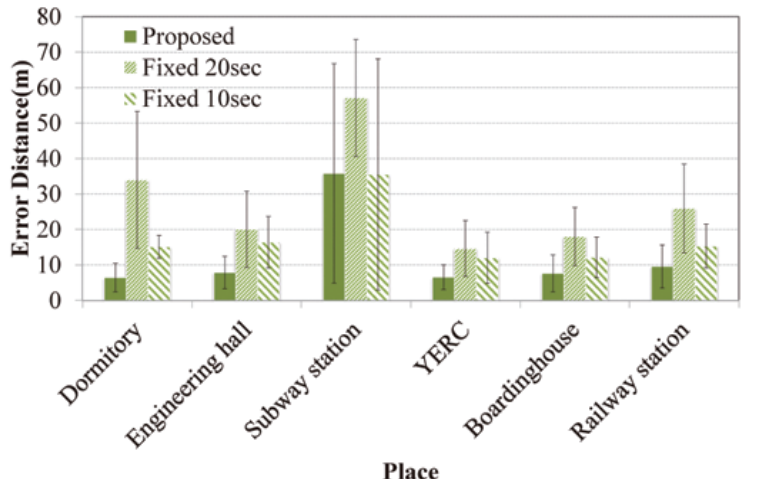

(b)

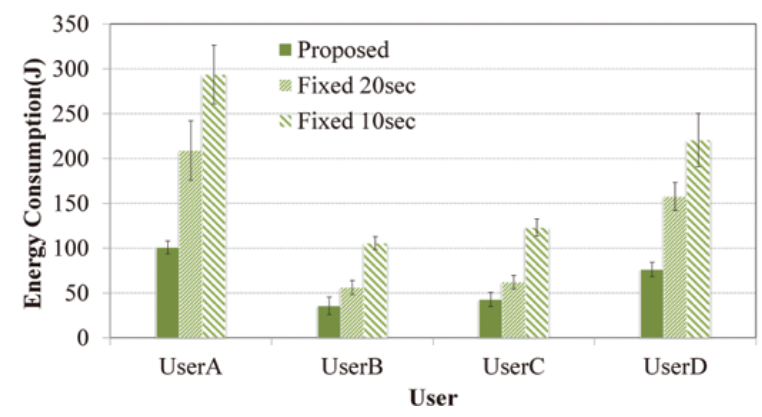

(c)

Fig. 15. Performance according to the handover method in the real-life experiment. (a) time error, (b) error distance, and (c) energy consumption.

in the evening. Participant B departed for the Yonsei Engineering Research Center (YERC) from the subway station in the morning, and vice versa in the evening. Participant $C$ walked to the Engineering Hall from his boarding house. Participant $\mathrm{D}$ walked to the YERC from the railway station. The path lengths of participant A, B, C, and D were $1.8 \mathrm{~km}, 0.7 \mathrm{~km}, 0.6 \mathrm{~km}$, and $0.7 \mathrm{~km}$, respectively.

Figure 15(a) shows the time error between the handover point and the time when the user entered the indoor environment. The proposed algorithm showed a time error of 2-3 seconds, while fixed $20 \mathrm{sec}$ and fixed $10 \mathrm{sec}$ showed errors of 10-15 seconds and 4-5 seconds, respectively. This result is natural, as the proposed algorithm performs SNR monitoring near the entrance after several visits. As shown in Fig. 15(b), the error distances of the proposed algorithm were $6.4 \mathrm{~m}, 7.9 \mathrm{~m}, 35.8 \mathrm{~m}, 6.5 \mathrm{~m}, 7.6 \mathrm{~m}$, and $9.5 \mathrm{~m}$ at the dormitory, Engineering Hall, subway station, YERC, Boardinghouse, and railway station, respectively, which were less than those of fixed $20 \mathrm{sec}$ and fixed $10 \mathrm{sec}$. The error distance of the handover point fluctuated depending on the place, although the time error was very small regardless of the place. This is because the handover location was decided by using GPS location at the handover point. The proposed algorithm showed a large distance error of $35.8 \mathrm{~m}$ at the subway station, because GPS accuracy is very low there due to the high-rise buildings around the station. Average energy consumption during the experiment is shown in Fig. 15(c). The proposed algorithm always consumed less energy than the others. Moreover, the efficiency of the proposed algorithm improved when the participant walked a longer path. Participant A consumed more energy than others since she walked a longer path. However, energy consumption of the proposed algorithm does not increase proportionally to the path length, as GPS was sampled in a low frequency outside the IB. On the other hand, the number of GPS samplings 


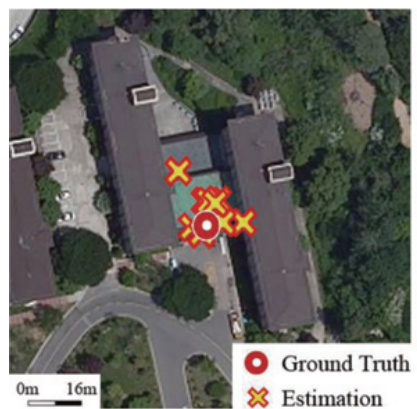

(a)

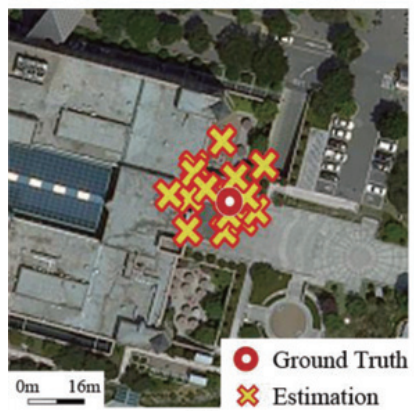

(d)

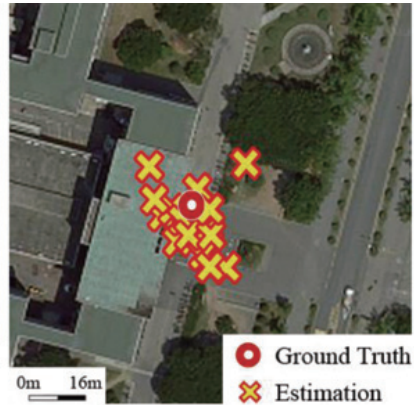

(b)

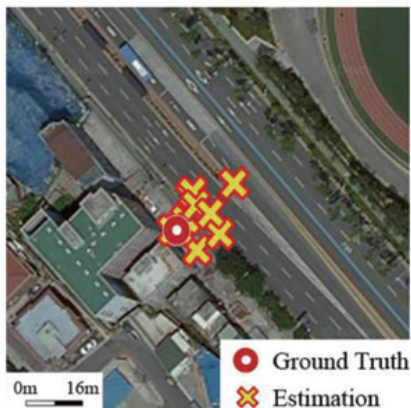

(e)

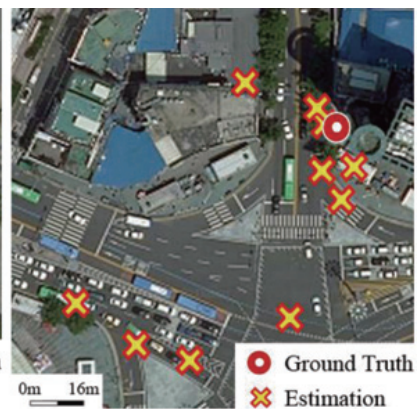

(c)

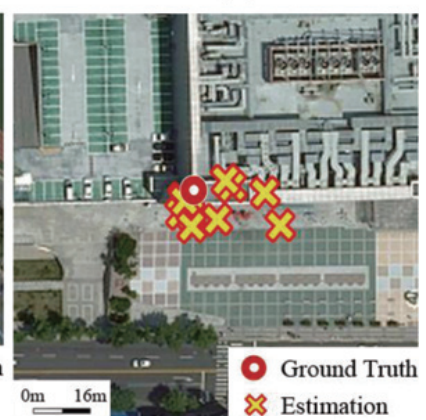

(f)

Fig. 16. Estimated locations of the entrance in the real-life experiment. (a) Domitory, (b) Engineering Hall, (c) Subway Station, (d) Research Center, (e) Boardinghouse, and (f) Railway station.

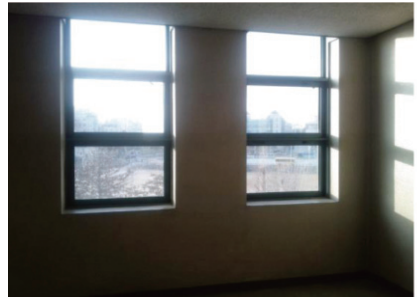

(a)

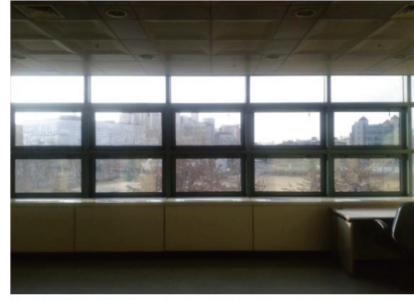

(b)

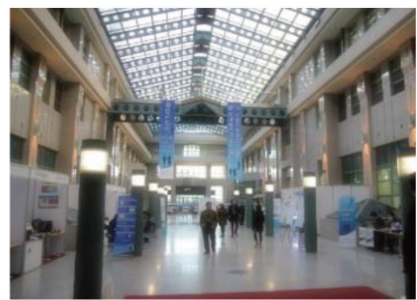

(c)

Fig. 17. Indoor places where the SNR is abnormally high. (a) near a small window, (b) near a big window, and (c) under a glass roof.

increased linearly as the path became longer with the fixed sampling interval.

Figure 16 illustrates the distance between the handover point and the building entrances. Except for the subway station, where the GPS accuracy is too low, most of the handover points were close to the building entrances. This means that the proposed algorithm can be exploited for finding building entrances, which is useful for location-based services.

\subsection{Indoor-to-outdoor handover}

The proposed system focused on handover, specifically from outdoor to indoor, although handover from indoor to outdoor is also important. The system should switch back to GPS when a user emerges from a building. This can be done by periodic GPS sampling; handover is performed when the SNR 


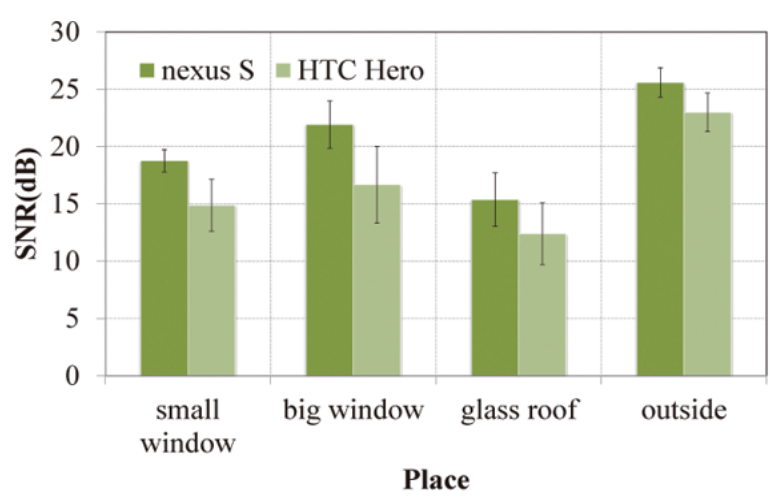

(a)

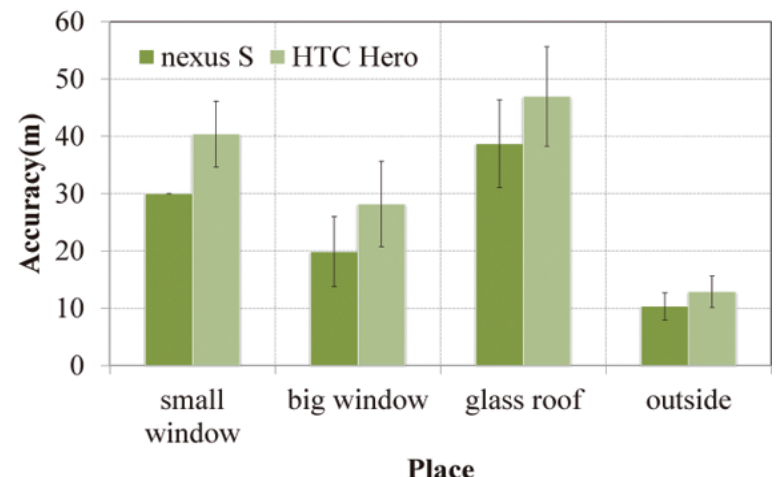

(b)

Fig. 18. comparison of (a) SNR and (b) GPS accuracy in the indoor places with those in outdoor environment.

level reaches a certain level where the user is considered to be in outdoors. However, it is difficult to determine the SNR level for the handover since the SNR level is abnormally high near the windows even in the building. Hence, we conducted an experiment that measures the SNR with Nexus S and HTC Hero in three different indoor places: near small windows, near big windows, and under a glass roof, as shown in Fig. 17. Figure 18(a) compares the SNR in the indoor places with that of outside the building. The SNR near the big windows was the highest among indoor places, and the value was similar with the SNR in outdoor. Moreover, the SNR level varied depending on the device type. The result showed that using SNR level is not adequate for handover from indoor to outdoor. Figure 18(b) shows the accuracy information acquired from GPS. Unlike the SNR level, we see the obvious differences in the accuracy of indoor and outdoor environment; the difference between near the big window and outside was about $10 \mathrm{~m}$ regardless of devices. The experimental result shows that the handover from indoor to outdoor can be done accurately by using a fixed threshold in the GPS accuracy.

\section{Conclusion}

In this paper, we proposed an accurate handover technique that monitors SNR changes of specific GPS satellites. The proposed system performs handover exactly at the entrance by selecting satellites that are positioned behind the user, at high elevations. The proposed algorithm has the additional advantage of finding the location of the entrance, which can be exploited by many location-based services. We also proposed a method that reduces energy consumption by increasing the sampling rate gradually while visiting POIs repetitively. The experiment results demonstrate that the proposed algorithm can find the location of a building entrance as the handover point within a few visits and with little energy consumption.

In our future work, we plan to overcome the requirement of revisits to a place by cooperating with other users. A user can determine a handover point accurately even at a first visit by using IB and DP of other users. We also plan to implement a life-logging system that uses the proposed handover technique. The system tracks the user with GPS outdoors and with the PDR method indoors. The location error of the POI in indoor environments is expected to decrease with a precise handover. We consider the collaboration of anonymous users to find the location of the entrance to an arbitrary building. Security issues and attracting voluntary participation from anonymous users should be also considered. 


\section{Acknowledgments}

This work was supported by the National Research Foundation of Korea (NRF) grant funded by the Korean government, Ministry of Education, Science and Technology under Grant No. 2012-0005522.

\section{References}

[1] J. Chon and H. Cha, Lifemap: A smartphone-based context provider for location-based services. Pervasive Computing, IEEE 10(2) (2011), 58-67.

[2] Inc. Eye-Fi. What is geotagging? http://www.eye.fi/how-it-works/features/geotagging, Accessed 20 February 2012.

[3] S.R. Gulliver, G. Ghinea, M. Patel and T. Serif, A context-aware tour guide: User implications, Mobile Information Systems 3(2) (2007), 71-88.

[4] M. Safar, H. Sawwan, M. Taha and T. Al-Fadhli, Virtual social networks online and mobile systems, Mobile Information Systems 5(3) (2009), 233-253.

[5] Facebook. Explore facebook: Places. http://www.facebook.com/places/, Accessed 20 February 2012.

[6] M. Mohr, C. Edwards and B. McCarthy, A study of lbs accuracy in the uk and a novel approach to inferring the positioning technology employed, Computer Communications 31(6) (2008), 1148-1159.

[7] M. Chen, T. Sohn, D. Chmelev, D. Haehnel, J. Hightower, J. Hughes, A. LaMarca, F. Potter, I. Smith and A. Varshavsky, Practical metropolitan-scale positioning for gsm phones. UbiComp 2006: Ubiquitous Computing, 2006, pp. $225-242$.

[8] E.D. Kaplan and C.J. Hegarty, Understanding GPS: principles and applications. Artech House Publishers, 2006.

[9] Ubisence. Ubisense real-time location systems. http://www.ubisense.net/en/rtls-solutions, Accessed 20 February 2012.

[10] M. Muñoz-Organero, P.J. Muñoz-Merino and C. Delgado Kloos, Using bluetooth to implement a pervasive indoor positioning system with minimal requirements at the application level, Mobile Information Systems 8(1) (2012), 73-82.

[11] P. Bahl and V.N. Padmanabhan, Radar: An in-building rf-based user location and tracking system. In INFOCOM 2000. Nineteenth Annual Joint Conference of the IEEE Computer and Communications Societies. Proceedings, IEEE 2 (2000), pp. 775-784.

[12] A. LaMarca, Y. Chawathe, S. Consolvo, J. Hightower, I. Smith, J. Scott, T. Sohn, J. Howard, J. Hughes, F. Potter et al., Place lab: Device positioning using radio beacons in the wild. Pervasive Computing, 2005, pp. 301-306.

[13] O. Mezentsev, J. Collin and G. Lachapelle, Pedestrian dead reckoningaCa solution to navigation in gps signal degraded areas? Geomatica 59(2) (2005), 175-182.

[14] I. Constandache, R.R. Choudhury and I. Rhee, Towards mobile phone localization without war-driving. In INFOCOM, 2010 Proceedings IEEE, 2010, pp. 1-9.

[15] S. Beauregard and H. Haas, Pedestrian dead reckoning: A basis for personal positioning. In Proceedings of the 3rd Workshop on Positioning, Navigation and Communication (WPNC'06), 2006, pp. 27-35.

[16] M. Kourogi, N. Sakata, T. Okuma and T. Kurata, Indoor/outdoor pedestrian navigation with an embedded gps/rfid/selfcontained sensor system. Advances in Artificial Reality and Tele-Existence, 2006, pp. 1310-1321.

[17] Skyhook Wireless. Skyhook, the worldwide leader in location positioning, context and intelligence. http://skyhookwire less.com/, Accessed 20 February 2012.

[18] M. Tanigawa, J.D. Hol, F. Dijkstra, H.J. Luinge and P.J. Slycke, Augmentation of low-cost gps/mems ins with uwb positioning system for seamless outdoor/indoor positioning. ION GNSS, Savannah, Georgia, vol session C, 4, 2004.

[19] D.S. Chiu and K.P. O'Keefe, Seamless outdoor-to-indoor pedestrian navigation using gps and uwb. In Proceedings of the 21st International Technical Meeting of the Satellite Division of the Institute of Navigation (ION GNSS 2008), The Institute of Navigation 1 (2008), 322-333.

[20] R. Hansen, R. Wind, C.S. Jensen and B. Thomsen, Seamless indoor/outdoor positioning handover for location-based services in streamspin. In Mobile Data Management: Systems, Services and Middleware, 2009. MDM'09. Tenth International Conference on, IEEE, 2009, pp. 267-272.

[21] S. Gaonkar, J. Li, R.R. Choudhury, L. Cox and A. Schmidt, Micro-blog: sharing and querying content through mobile phones and social participation. In Proceeding of the 6th international conference on Mobile systems, applications, and services, ACM, 2008, pp. 174-186.

[22] M.B. Kjærgaard, J. Langdal, T. Godsk and T. Toftkjær, Entracked: energy-efficient robust position tracking for mobile devices. In Proceedings of the 7th international conference on Mobile systems, applications, and services, ACM, 2009, pp. 221-234.

[23] I. Constandache, S. Gaonkar, M. Sayler, R.R. Choudhury and L. Cox, Energy-efficient localization via personal mobility profiling. Proceedings of MobiCase, 2009. 
[24] Y. Chon, E. Talipov, H. Shin and H. Cha, Mobility prediction-based smartphone energy optimization for everyday location monitoring. In Proceedings of the 9th ACM Conference on Embedded Networked Sensor Systems (SenSys 2011), ACM, 2011, pp. 82-95.

Yungeun Kim is currently a Ph.D. candidate at the Department of Computer Science, Yonsei University, Korea. He received his B.Sc. degree in computer science from Yonsei University in 2008. His research interests include mobile and ubiquitous computing, and smartphone-based localization system.

Songhee Lee is an employee at the department of Home Appliances, Samsung Electronics, Korea. She received her B.Sc.degree in Kyunghee University in 2006. She received her M.Sc degree in computer science from Yonsei University in 2011. Her research interests include mobile and ubiquitous computing and convergence system.

Sukjun Lee is a M.Sc. student at the Department of Computer Science, Yonsei University, Korea. He received his B.Sc. degree in computer science from Yonsei University in 2011. His research interests include mobile and ubiquitous computing, context-aware computing and green computing.

Hojung Cha is currently a professor in computer science at Yonsei University, Seoul, Korea. His research interests include wireless and mobile systems, embedded operating systems and sensor network systems. He received his B.S. and M.S. in computer engineering from Seoul National University, Korea, in 1985 and 1987, respectively. He received his Ph.D. in computer science from the University of Manchester, England, in 1991. 

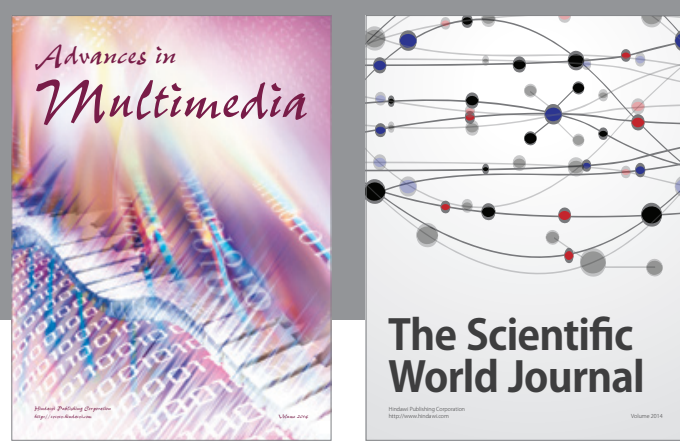

The Scientific World Journal
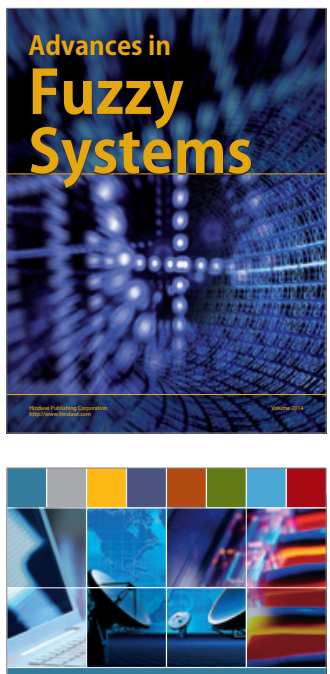

Computer Networks and Communications
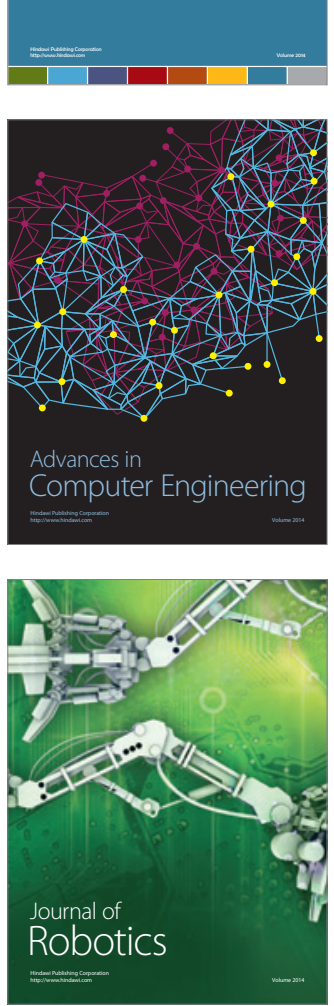
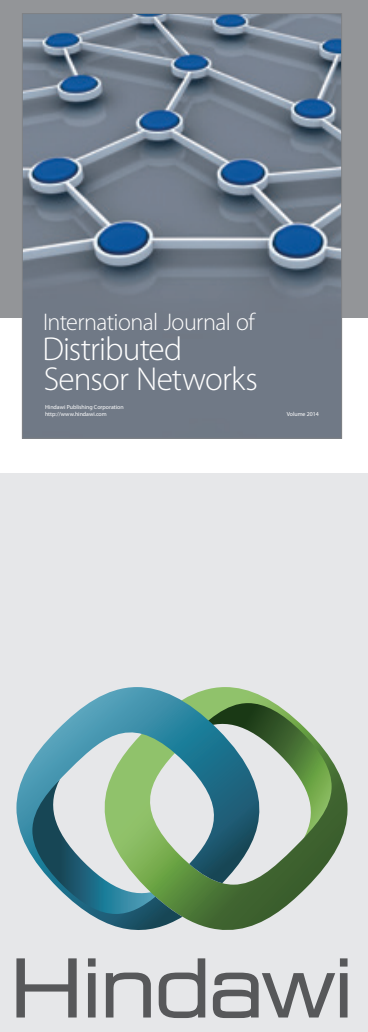

Submit your manuscripts at

http://www.hindawi.com
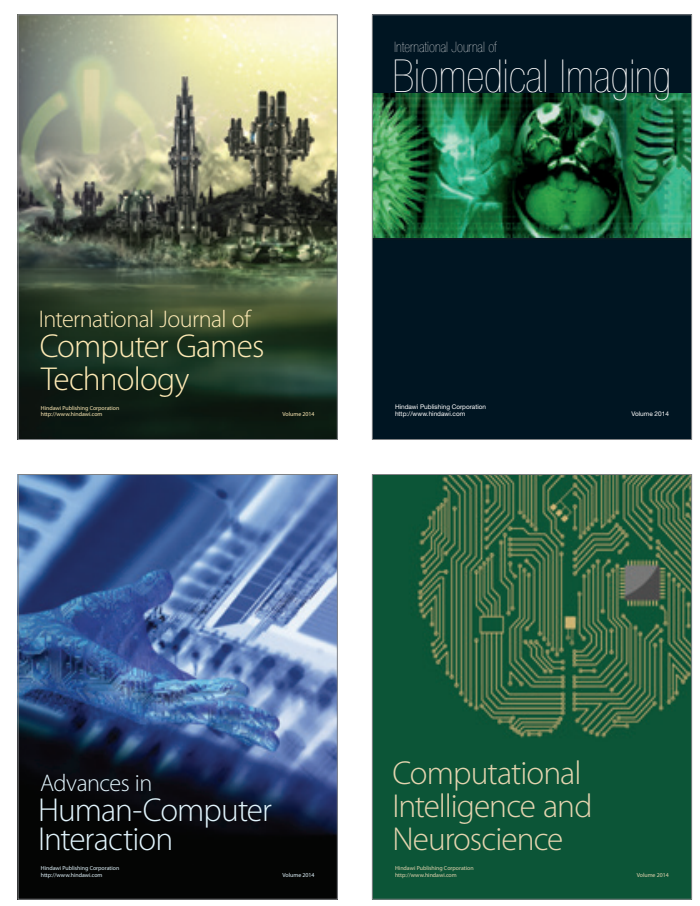
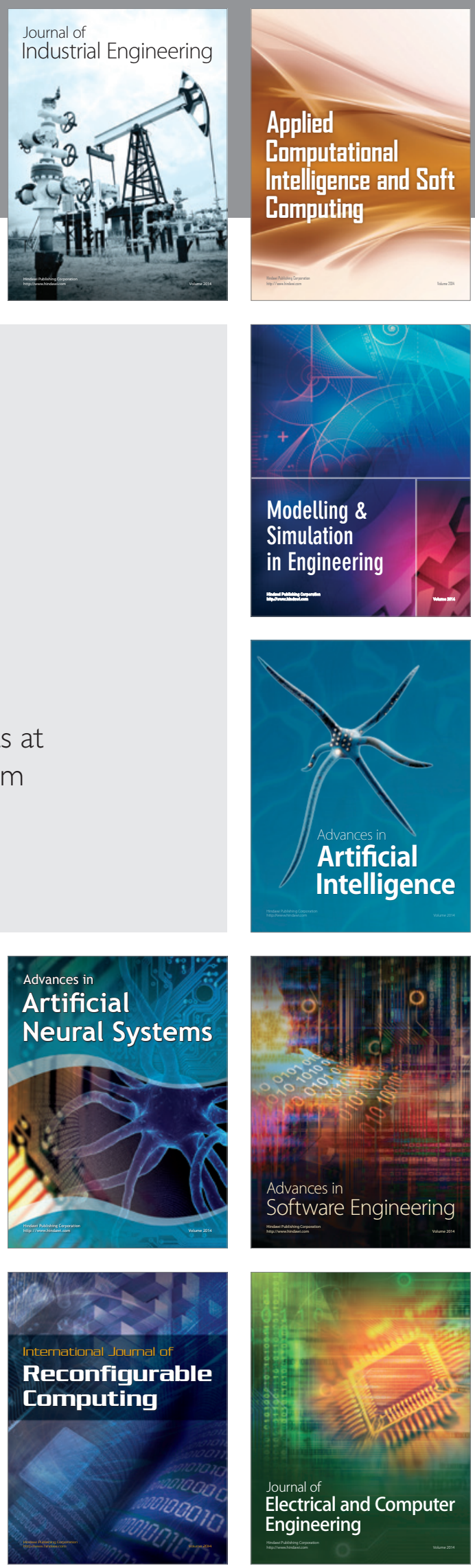